\title{
Non-small cell lung cancer clinical management patterns in the Middle East and North Africa region
}

\section{Introduction}

Lung cancer is the most common cancer worldwide. In 2013, lung cancer accounted for $13 \%$ of newly diagnosed cancer cases Ferlay J et al. ${ }^{1}$ It is also the leading cause of cancer death in the world Jemal A et al., ${ }^{2}$ Of the 7.3 million deaths caused by cancer every year, $17 \%$ were due to lung cancer Ferlay $\mathrm{J}$ et al. ${ }^{3}$ The majority of cases occur in the less developed countries (58\%) (Ferlay et al, 2014, IARC). Moreover, lung cancer is a top leader among males, yet in females incidence rates are generally lower Ferlay et al., ${ }^{1}$ Worldwide, lung cancer in females was rated as the third most frequent cancer type ( $8.8 \%$ of all cancers) $)^{4}$ and the second most common cause of cancer death (12.8\%) Ferlay et al. 5

In the Middle East and North Africa (MENA) region, lung cancer is also the most prevalent cancer in men, with an age-standardized incidence of $15.9 \%$ and an $8.2 \%$ mortality rate. ${ }^{4}$ Available data on cancer burden indicates that lung cancer rates in the Arab region will continue to grow due to several factors such as changes towards westernised lifestyles, population growth, increased smoking prevalence and aging Salim et al. ${ }^{6}$ Population forecasts in the Arab region, estimated that from 2008 to 2020, new lung cancer cases in both sexes will increase from 9537 to 14,788 and 7059 to 14,788 for those aged below and above 65 respectively Ferlay et al. ${ }^{5}$

The burden caused by this disease in the region is further intensified by the fact that resources available for obtaining correct diagnosis and tumor node metastasis (TNM) classification differ throughout centres in the MENA region, and are exemplified by the lack of resources for Positron Emission Tomography (PET) examination in many sites. Thus, histopathologically confirmed lung cancer is essential for optimal management of the individual patient and must be secured before chemotherapy and radiotherapy are initiated. However, in rare cases it may be impossible to obtain tumor tissue for examination due to multiple significant co-morbidities or poor performance status. There is also a huge variation in how novel treatments are adopted and implemented among different countries and even among different regions in the same country. When discussing these differences in the management of lung cancer patients, it should be emphasized that we only have limited knowledge of the real magnitude of these national and regional variations.

Currently, most investigation into lung cancer and specifically nonsmall cell lung cancer (NSCLC) is focused on the clinical outcomes of some treatment strategies and, in the field of epidemiology, in assessing the likely risk factors associated with the disease. There are only a few current studies on how patients are managed in real life practice Fervers B et al., ${ }^{7}$ Bren L, ${ }^{8}$ Atherton PJ,${ }^{9}$ LoRusso PM et al., ${ }^{10}$ Saint Jacques $\mathrm{N}$ et al., ${ }^{11}$ these are clinical epidemiology studies. In view of this it would be very useful for physicians to be informed of treatment strategies used around the MENA region and to also have information on the impact of these treatment options on overall costs and patient outcomes, in order to determine the best management pattern for each type of patient.

The lack of good, updated, organized and accessible local epidemiological data, clinical management data and knowledge of how to use healthcare resources in view of the many different
Volume 9 Issue 4 - 2018

\author{
Mohammed Jaloudi,' Kakil Ibrahim Rasul, ${ }^{2}$ \\ Farid Khalifa, ${ }^{3}$ Ravi Patnaik, ${ }^{4}$ Jihad Kanbar, \\ Mohamed Al-Sayed, ${ }^{6}$ Amgad $\mathrm{Kamal}^{7}$ \\ 'Tawam hospital, UAE \\ ${ }^{2}$ Hamad Medical Corporation, Qatar \\ ${ }^{3}$ Noor Hospital, Bahrain \\ ${ }^{4}$ Brunei Cancer Center, Brunei \\ ${ }^{5}$ Tawam hospital, UAE \\ ${ }^{6,7}$ AstraZeneca, UK
}

Correspondence: Kakil Ibrahim Rasul, Senior Consultatnt, Haematology/Oncology National Center for Cancer Care and Research Doha, Qatar, Tel 974-5-5876832, Email kakil1954@gmail.com

Received: July 09, 2018 | Published: July II, 2018

treatment regimens available, all leads to an underestimation of the actual burden of lung cancer and associated unmet medical needs, with consequences for payers and health decision makers, at the national and the MENA region level, as well as for patients and society. For this reason, this study was designed to provide information on the clinical effectiveness of existing products, health care resources that are associated with existing therapy and information on patients' quality of life in the MENA region. It is also the first study to assess NSCLC patient treatment patterns in the MENA region.

\section{Methods}

\section{Sample}

To ensure a valid picture of the real life management of NSCLC patients, sites selected focused on representative institutions (hospitals or centers) where lung cancer patients were being managed, in all participating countries. Initially, institutions from the United Arab Emirates (UAE), Qatar, Bahrain, Oman and Kuwait containing large populations of NSCLC patients were targeted to participate in the study. Kuwait and Oman had initially declined to participate. A total of four centers; 2 from UAE, 1 from Bahrain and 1 from Qatar accepted to participate in the study. One of the participating centers in the UAE was unable recruit participants for the study and eventually dropped out from the study. The three centers that participated in the study and were able to recruit subjects for the study were; Hamad Medical Corporation (HMC) Al-Amal Hospital, Qatar; Salmaniya Medical Complex, Bahrain; Tawam Hospital, UAE. Institutional Review Board (IRB) approvals were obtained from all participating centers respectively (HMC Ethics Committee, EC/IRB OncologyHematology Department and Al Ain Medical District Human Research Ethics Committee).

Patients with a confirmed NSCLC diagnosis (all stages), aged 18 or above, attending the responsible department of treating this type of disease for the first time at the participating sites from the first of February 2012 to the end of February 2015 were considered eligible. Patients were then informed about the study and were asked to sign an informed consent form which specified that there will be no change in 
the clinical management of their disease due to their enrolment in the study. Patients were followed up for a period of 12 months, and data on each patient was reported in a case report form that was completed at each evaluation visit. Information on each visit and any other relevant data related to any intermediate visits between the last evaluation visit and the first was reported (a maximum of 5 visits were reported).

\section{Measures}

The data collected in this study was solely based on patient's medical records at each evaluation visit as part of their routine clinical practice. Data collected included patient demographics (age, gender, smoking status (non-smoker, ex-smoker, habitual smoker)), co-morbidities and medical history, descriptive on the disease (symptoms, location, stage, TNM classification, histology, tumor grade (well differentiated (G1), moderately differentiated (G2), poorly differentiated (G3), undifferentiated (G4), un-assessable (GX)), patient outcomes (performance status (PS 0-4) and disease progression), treatments received (chemotherapy, radiotherapy, and surgery) and tests performed (X-rays, Computed tomography (CT) Scans, MRI, PET, Biomarkers), disease related adverse events (AEs) and therapy related AEs. After patients consented to participate in the study, they were then administered the Functional Status Questionnaire (FACT-L)

The FACT-L Erratum, ${ }^{12}$ Jette AM et al., ${ }^{13}$ measures 4 well-being subscales (physical, social/family, emotional and functional) and an additional section on concerns that includes items such as losing weight, breathing easy etc. Each subscale contains a number of items that are scored from 0-4 (0 "not at all"- 4"very much").

\section{Statistical analysis}

Descriptive statistics that include frequency tables were conducted to describe the profiles of our NSCLC study population, descriptives on the disease, the clinical management, clinical outcomes and the use of health care resources. Changes in FACT-L scores from baseline to the end of the study (Visit 5) were analyzed using ANCOVA models (GLM Univariate Model) to detect any improvements in patient's well-being. Finally, bivariate logistic regression models were built to assess the association of patient characteristics (including age, gender, smoking status, histology and performance status) with clinical outcome variables (mortality, developing treatment related events and clinical evaluation methods used (clinical/RECIST criteria). Model-based point estimates of odds ratios and corresponding $95 \%$ confidence intervals were reported. Analyses were carried out using SPSS 22.0. Significance for all statistical procedures were considered at $\mathrm{P}$-value $<0.05$.

\section{Results \\ Demographics and baseline characteristics}

A total of 25 NSCLC patients were recruited in the study at baseline (7/25 Bahrain; 11/25 UAE; 7/25Qatar). Only 16 of 25 patients completed the study; 3 dropped out and 6 patients died due to their disease. The mean age of the sample was $56.9( \pm 9.51)$ yrs, with $80 \%$ males. $28 \%(n=7)$ of our sample originated from GCC countries and $72 \%(\mathrm{n}=18)$ from non-GCC countries. Moreover, $56 \%$ were exsmokers, $32 \%$ non-smokers and $12 \%$ habitual smokers. 19 of the 25 patients reported data on having at least one relevant chronic condition other than lung cancer. $36.8 \%$ (7/19) reported having no other chronic condition, and $63.2 \%(12 / 19)$ reported hypertension.

\section{Disease related characteristics at baseline}

The most common symptoms reported at first visit were cough $(80 \%)$, dyspnoea (60\%), weight loss $(44 \%)$ and thoracic pain $(40 \%)$. $72 \%$ of the sample had a stage IV of NSCLC. $60 \%$ had a performance status of PS1, 24\% PS $0,8 \%$ PS 2 and $8 \%$ PS3. More than half had the upper right lobe as their primary tumor location (57.1\%), followed by the upper left lobe (23.8\%). Moreover, the most common histology type reported was that of adenocarcinoma $(70.8 \%)$, followed by squamous cell carcinoma (12.5\%), adenocarcinoma bronchioloalveolar $(8.3 \%)$ and adenosquamous carcinoma $(8.3 \%)$. None reported having large cell carcinoma. $39.1 \%, 21.7 \%, 21.7 \%, 13 \%$ and $4.3 \%$ had tumor grades of GX, G2, G3, G1 and G4 respectively. Within TNM classifications, $11 / 25$ had a T4 classification, 12/24 had an N3 classification and 15/23 had an M1 TNM classification (Table 1). The mean number of primary lesions in our sample was $3.13( \pm 3.31$ ), ranging between a minimum of 0 lesions to a maximum of 16 lesions. The lesions metastatic locations were mostly reported to be in the bone and locomotor (44\%), pleural effusion (32\%), respiratory (32\%) and brain (28\%).

Table I Disease-related characteristics of patients

\begin{tabular}{|c|c|c|}
\hline & $\mathbf{N}$ & $\%$ \\
\hline \multicolumn{3}{|l|}{ Stage of disease $(n=25)$} \\
\hline IIA & 1 & 4 \\
\hline IIIA & 1 & 4 \\
\hline IIIB & 5 & 20 \\
\hline IV & 18 & 72 \\
\hline \multicolumn{3}{|l|}{ Performance status $(n=25)$} \\
\hline PS 0 & 6 & 24 \\
\hline PS I & 15 & 60 \\
\hline PS 2 & 2 & 8 \\
\hline PS 3 & 2 & 8 \\
\hline PS 4 & 0 & 0 \\
\hline \multicolumn{3}{|l|}{ TNM classification } \\
\hline TI & 2 & 8 \\
\hline $\mathrm{T} 2$ & 4 & 16 \\
\hline T3 & 4 & 16 \\
\hline T4 & 11 & 44 \\
\hline TX & 4 & 16 \\
\hline Noa & 3 & 12 \\
\hline $\mathrm{NI}$ & 1 & 4 \\
\hline N2 & 8 & 32 \\
\hline N3 & 12 & 48 \\
\hline MOb & 7 & 28 \\
\hline MI & 16 & 64 \\
\hline \multicolumn{3}{|l|}{ Histology Type $(n=24)$} \\
\hline Adenocarcinoma & 17 & 70.8 \\
\hline Adenocarcinoma bronchioloalveolar & 2 & 8.3 \\
\hline Adenosquamous carcinoma & 2 & 8.3 \\
\hline Squamous cell carcinoma & 3 & 12.5 \\
\hline
\end{tabular}


Table continued.

\begin{tabular}{lll}
\hline & $\mathbf{N}$ & $\%$ \\
\hline Large cell carcinoma & 0 & 0 \\
Tumor grade $(\mathrm{N}=23)$ & 5 & 21.7 \\
Moderately differentiated $(\mathrm{g} 2)$ & 5 & 21.7 \\
Poorly differentiated $(\mathrm{g} 3)$ & 9 & 39.1 \\
Unassessable $(\mathrm{gx})$ & $\mathrm{I}$ & 4.3 \\
Undifferentiated $(\mathrm{g} 4)$ & 3 & 13 \\
Well differentiated $(\mathrm{gl})$ & & \\
Primary Tumor location $(\mathrm{N}=2 \mathrm{I})$ & $\mathrm{I}$ & 4.8 \\
Lower left lobe & 2 & 9.5 \\
Lower right lobe & $\mathrm{I}$ & 4.8 \\
Medium right lobe & 5 & 23.8 \\
Upper left lobe & $\mathrm{I}$ & 57.1 \\
Upper right lobe & & \\
\hline
\end{tabular}

${ }^{\text {a Total }} \mathrm{N}=24{ }^{\mathrm{b}}$ Total $\mathrm{N}=23$

\section{Treatment}

Imaging techniques for detection purposes were performed by all 25 patients. The most common were X-rays (88\%) and CT scans (84\%). Other techniques included; MRI (44\%), PET (48\%) and biomarkers $(26 \%)$. The most common reason for scheduled and non-scheduled visits of patients throughout the study period was for chemotherapy (Table 2). Only 4 of 25 patients came for radiotherapy at the second visit and 21/25 came for chemotherapy. First line chemotherapy was most commonly used in the treatment of NSCLC patients across all visits (Figure 1). NSCLC patients that received chemotherapy were also assessed on disease response. From the total number of visits ( $\mathrm{N}=111)$; $50 \%$ did not have a determined disease response, $20.8 \%$ reported the disease as stable, $13.9 \%$ reported the disease to be "progressing", 9.7\% reported "partial response" and $5.6 \%$ did not provide any data on disease response. Furthermore, the method used to evaluate patient's response to treatment was noted. Clinical assessments (75\%) were more commonly used as an evaluation criterion in comparison to the RECIST criteria.

Table 2 Reasons for scheduled and non-scheduled visits to participating site

\begin{tabular}{|c|c|c|c|c|c|c|c|c|}
\hline & \multicolumn{8}{|c|}{ Visit number } \\
\hline & \multicolumn{2}{|c|}{$*$ Visit $2(n=25)$} & \multicolumn{2}{|c|}{ Visit $3(n=24)$} & \multicolumn{2}{|c|}{ Visit $4(n=21)$} & \multicolumn{2}{|c|}{ Visit 5(n=16) } \\
\hline & $\mathbf{N}$ & $\%$ & $\mathbf{N}$ & $\%$ & $\mathbf{N}$ & $\%$ & $\mathbf{N}$ & $\%$ \\
\hline \multicolumn{9}{|l|}{ Reasons } \\
\hline Follow up & 2 & 8 & 3 & 12.5 & 0 & 0 & I & 6.3 \\
\hline Chemotherapy & 21 & 84 & 18 & 75 & 15 & 71.4 & 12 & 75 \\
\hline Radiotherapy & 4 & 16 & 0 & 0 & 0 & 0 & 0 & 0 \\
\hline Other & 1 & 4 & 3 & 12.5 & 6 & 28.6 & 3 & 18.8 \\
\hline
\end{tabular}

*Visit 2, the \%'s are not mutually exclusive

*Visit 2, the \%'s are not mutually exclusive

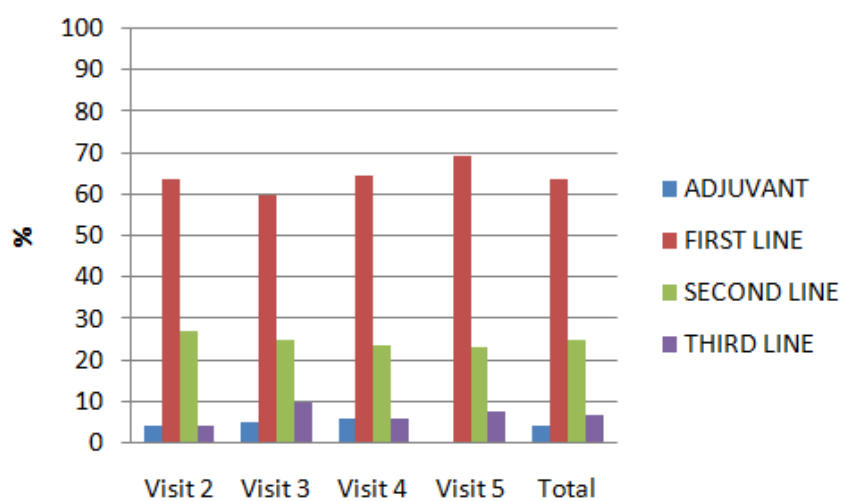

Figure I Chemotherapy Lines Used.

Visit $2(N=22)$; Visit $3(N=20)$;Visit $4(N=17)$;Visit $5(N=\mid 3)$, Total $(N=72)$

\section{Treatment related AEs}

Not all NSCLC patients that were undergoing chemotherapy reported on the number of AEs experienced due to treatment. Among those who did, 5 of 11 patients reported at least one AE at visit 2, 4 of 7 reported at least one $\mathrm{AE}$ at visit 3, 3 of 7 reported at least one $\mathrm{AE}$ at visit 4 and 2 of 4 reported at least one AE at visit 5 (Figure 2). Severe AEs were also reported. None reported severe AEs at visit 2 and 3. 1 of 7 patients and 2 of 4 patients reported having a severe $\mathrm{AE}$ at visit 4 and 5 respectively. Due to these AEs, $11.6 \%$ reported a change in their NSCLC treatment; $50 \%$ specified treatment delay, $40 \%$ permanent interruption, $10 \%$ did not provide data.

\section{Functional Well-being}

Scores on all 5 FACT-L well-being subscales decreased across the study period (Table 3$)$. This decrease was only significant $(p: 0.013)$ for physical well-being scores $[13.0( \pm 6.01)$ to $7.55( \pm 3.91)]$ and borderline $(p: 0.097)$ for emotional well-being scores $[10.33( \pm 6.34)$ to $6.70( \pm 2.62)]$. 


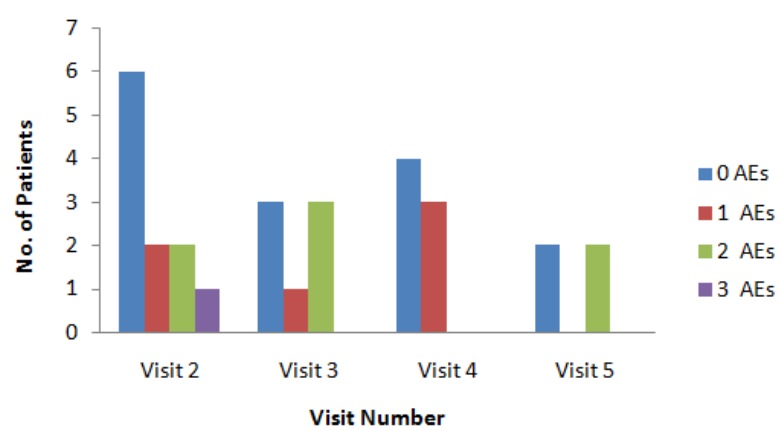

Figure 2 The number of adverse events experienced by patients due to receiving chemotherapy.

Visit $2(\mathrm{~N}=$ I I); Visit $3(\mathrm{~N}=7)$; Visit $4(\mathrm{~N}=7)$; Visit $5(\mathrm{~N}=4)$

Table 3 Changes in FACT-L well being scores across study period

\begin{tabular}{|c|c|c|c|c|c|}
\hline $\begin{array}{l}\text { Well-being type } \\
\text { (FACT-L) }\end{array}$ & Visit \# & Visit (N) & $\begin{array}{l}\text { Mean } \\
\text { score }\end{array}$ & SD & p-value \\
\hline $\begin{array}{l}\text { Physical Well- } \\
\text { Being }\end{array}$ & I & 18 & 13 & 6.01 & $0.013^{* *}$ \\
\hline $\begin{array}{l}\text { (Higher score; } \\
\text { Worst) }\end{array}$ & 5 & II & 7.55 & 3.91 & \\
\hline $\begin{array}{l}\text { Social/Family Well- } \\
\text { Being }\end{array}$ & I & 18 & 20.78 & 5.75 & 0.202 \\
\hline $\begin{array}{l}\text { (Higher score; } \\
\text { Better) }\end{array}$ & 5 & 10 & 17.6 & 6.87 & \\
\hline $\begin{array}{l}\text { Emotional Well- } \\
\text { Being }\end{array}$ & I & 18 & 10.33 & 6.343 & $0.097 *$ \\
\hline $\begin{array}{l}\text { (Higher score; } \\
\text { Worst) }\end{array}$ & 5 & 10 & 6.7 & 2.627 & \\
\hline $\begin{array}{l}\text { Functional Well- } \\
\text { Being }\end{array}$ & I & 18 & 16.83 & 5.618 & 0.789 \\
\hline $\begin{array}{l}\text { (Higher score; } \\
\text { Better) }\end{array}$ & 5 & II & 16.27 & 5.101 & \\
\hline $\begin{array}{l}\text { Additional } \\
\text { Concerns }\end{array}$ & I & 18 & 12.22 & 5.65 & 0.189 \\
\hline $\begin{array}{l}\text { (Higher score; } \\
\text { Worst) }\end{array}$ & 5 & 10 & 9.5 & 3.89 & \\
\hline
\end{tabular}

Additional concerns includes: short of breath, loss of weight, clear thoughts, coughing, hair loss, appetite, tightness in chest, easy breathing

$* *_{p}<0.05$

$*_{p}<0.1$ (borderline significance)

\section{Predictors of treatment related AES, Recist and clinical criteria}

Having a performance status of 3 , was significantly associated with developing a treatment related event ( $p: 0.03)$, whereby the odds of having a treatment related event within individuals with a performance status of 3 is 32times greater than individuals with a performance status 0 . There is also a trend towards significance ( $p$-value: 0.089 ) for ex-smokers to have a 6.4 times more risk of developing a treatment related event when compared to non-smokers.
Gender, smoking status, and histology were significantly associated with using RECIST and clinical criteria for evaluation purposes respectively. NSCLC patients that were males (OR:0.14; $p: 0.014)$ and ex-smokers (OR:0.01; p:0.019) were less likely going to have an evaluation based on RECIST criteria (when compared to females and non-smokers), yet they were more likely going to have an evaluation based on a clinical criterion (OR:4.73, p:0.037 ; OR:13.14, $p: 0.021)$. Moreover, patients with a histology of adenocarcinoma bronchioloalveolar had greater odds (OR:1.32, p:0.03) of using RECIST criteria, whilst patients with a histology of adenocarcinoma had a trend towards significance of having greater odds (OR:7.12, $p: 0.07$ ) of using clinical criteria for evaluation purposes (when compared to squamous cell carcinoma). Moreover, there is a trend towards significance that individuals with a performance status of 2 are less likely going to have an evaluation based on clinical criterion when compared to individuals with a performance status of 0 (OR:0.15, p:0.07). Detailed results are available upon request.

\section{Discussion}

This multi-national, multi-centre, non-interventional, prospective cohort-study provides new insights into treatment patterns of NSCLC patients in the MENA region. The study was able to fulfil its objectives of producing a descriptive analysis on the NSCLC population in the region and to provide real-life data on their clinical management and treatment patterns. Similar to what was found in the literature, the majority of the patients in our sample had locally advanced or disseminated disease at diagnosis ( $72 \%$ stage IV). The most common histology and primary tumor location for NSCLC patients in our sample was adenocarcinoma and the upper right lobe respectively. The most common symptoms at first visits of NSCLC patients were coughing, dyspnoea and weight loss.

$\mathrm{X}$-ray and CT-Scanners were used mostly as imaging techniques for NSCLC patients. The most common type of therapy used was chemotherapy, and specifically first line chemotherapy. At all visits, nearly half of the sample required 1day hospital admission for chemotherapy administration. Very few NSCLC patients had radiotherapy as their therapy technique. Moreover, among patients under chemotherapy, very few reported any sort of adverse event or severe adverse event. ${ }^{14}$

Patients' ratings of well-being improved across the study period and were significant for ratings of physical and emotional wellbeing (borderline for the latter). Having a performance status " 3 " is a risk for developing treatment related events when compared to PS0 (reference group). There is also a trend towards significance for ex-smokers to have a 6.4times more risk of developing a treatment related event when compared to non-smokers. NSCLC patients that are males, older age and ex-smokers are inversely associated with using RECIST criteria for judgement purposes. Having histology of adenocarcinoma bronchioloalveolar is significantly associated with RECIST criteria in the positive direction. Patients with adenocarcinoma bronchioloalveolar are more likely going to have RECIST criteria for evaluation purposes than patients with squamous cell carcinoma (OR:16).

\section{Limitations}

Since this study only included a sample of 25 NSCLC patients, the results are not representative of the whole NSCLC population in 
the MENA region. Larger scale epidemiological studies of this sort are further encouraged to determine effective and cost- effective treatments in the real world.

\section{Acknowledgements}

None.

\section{Conflict of interest}

Author declares that there is no conflict of interest.

\section{References}

1. Ferlay J, Soerjomataram I, Ervik M, et al. GLOBOCAN 2012 v1.0, Cancer Incidence and Mortality Worldwide: IARC CancerBase No. 11. Lyon, France: International Agency for Research on Cancer; 2014.

2. Jemal A, Bray F, Center MM, et al. Global cancer statistics. CA Cancer J Clin. 2011;61(2):69-90.

3. Ferlay J, Autier P, Boniol M, et al. Estimates of the cancer incidence and mortality in Europe in 2006. Annals of Oncology. 2007;18(3):581-592.

4. International Agency for Research on Cancer. GLOBOCAN 2012: Estimated Incidence, Mortality and Prevalence Worldwide in 2012. All Cancers (excluding non-melanoma skin cancer). 2012.

5. Ferlay J, Shin HR, Bray F, et al. GLOBOCAN 2008, Cancer Incidence and Mortality Worldwide: IARC CancerBase No. 10 [Internet]. Lyon, France: International Agency for Research on Cancer; 2010.
6. Salim EI, Jazieh AR, Moore MA. Lung cancer incidence in the Arab League Countries: Risk Factors and Control (review). Asian Pacific J Cancer Prev. 2011;12(1):17-34.

7. Fervers B, Remy-Stockinger M, Mazeau-Woynar V, et al. CoCanCPG. Coordination of cancer clinical practice in Europe. Tumori. 2008;94(2):154-9.

8. Bren L. The Importance of Patient-Reported Outcomes.. It's All About the Patients. FDA Consumer Magazine. 2006; 40(6):26-32.

9. Atherton PJ, Sloan JA. Rising importance of patient-reported outcomes. The lancet oncology. 2006;7(11):883-884.

10. LoRusso PM, Herbst RS, Rischin D, et al. Improvements in Quality of Life and Disease-related Symptoms in Phase I Trials of the Selective Oral Epidermal Growth Factor Receptor Tyrosine Kinase Inhibitor ZD1839 in Non-Small Cell Lung Cancer and Other Solid Tumours. Clin Cancer Research. 2003;9(6):2040-2048.

11. Saint-Jacques N, Rayson D, Al-Fayea T, et al. Waiting times in early-stage non-small cell lung cancer (NSCLC). J Thorac Oncol. 2008;3(8):865-70.

12. Erratum. J Gen Intern Med. 1986;1:427.

13. Jette AM, Davies AR, et al. The Functional Status Questionnaire: Reliability and validity when used in primary care. J Gen Intern Med. 1986;1(3):143-149.

14. Flanagan JC. Measurement of the quality of life: Current state of the art. Arch Phys Med Rehabil. 1982;63:56-59. 